\title{
Mechanical Design of the SMC (Short Model Coil) Dipole Magnet
}

\author{
F. Regis, P. Manil, P. Fessia, M. Bajko, and G. de Rijk
}

\begin{abstract}
The Short Model Coil (SMC) working group was set in February 2007 within the Next European Dipole (NED) program, in order to develop a short-scale model of a $\mathrm{Nb}_{3} \mathrm{Sn}$ dipole magnet. The SMC group comprises four laboratories: CERN/TE-MSC group (CH), CEA/IRFU (FR), RAL (UK) and LBNL (US). The SMC magnet was originally conceived to reach a peak field of about $13 \mathrm{~T}$ on conductor, using a $2500 \mathrm{~A} / \mathrm{mm}^{2}$ Powder-In-Tube (PIT) strand. The aim of this magnet device is to study the degradation of the magnetic properties of the $\mathrm{Nb}_{3} \mathrm{Sn}$ cable, by applying different level of pre-stress. To fully satisfy this purpose, a versatile and easy-to-assemble structure has to be realized. The design of the SMC magnet has been developed from an existing dipole magnet, the SD01, designed, built and tested at LBNL with support from CEA. In this paper we will describe the mechanical optimization of the dipole, starting from a conceptual configuration based on a former magnetic analysis. Two and three-dimensional Finite Element Method (FEM) models have been implemented in ANSYS and in CAST3M, aiming at setting the mechanical parameters of the dipole magnet structure, thus fulfilling the design constraints imposed by the materials.
\end{abstract}

Index Terms-High field magnets, mechanical modeling, superconducting magnet design.

\section{INTRODUCTION}

$\mathbf{S}$ EVERAL $\mathrm{Nb}_{3} \mathrm{Sn}$ magnets have been tested in the US since 1997 [1]-[4] within the High Field Magnets and LARP programs. In Europe, in January 2008, the HFM (High Field Magnets) program took over the NED program, which was mainly focused on the $\mathrm{Nb}_{3} \mathrm{Sn}$ cable development. The SMC is the first test dipole of the European HFM program using the $\mathrm{Nb}_{3} \mathrm{Sn}$ conductor. $\mathrm{Nb}_{3} \mathrm{Sn}$ magnets feature high magnetic fields involving large Lorentz forces $(\approx 2 \mathrm{MN} / \mathrm{m}$ for the SMC along the cable broad face), considerably higher than in Nb-Ti magnets [5]. Consequently, a proper pre-stress has to be set in order to prevent the coil from any motion which might lead to magnet quenches. On the other hand, this pre-stress has to respect what is considered as the $\mathrm{Nb}_{3} \mathrm{Sn}$ mechanical safe limit, i.e. $150 \mathrm{MPa}$ on the insulated coil block. Nevertheless this limit is not yet well understood, being mainly based on experimental evidence of magnets tests [5]. The aim of the SMC working group is to design a subscale dipole magnet where different levels of

Manuscript received October 19, 2009. First published March 18, 2010; current version published May 28, 2010.

F. Regis. P. Fessia, M. Bajko, and G. de Rijk are with CERN (European Organization for Nuclear Research) (e-mail: federico.regis@ cern.ch).

P. Manil is with CEA/Saclay, IRFU/SIS, 91191 Gif-sur-Yvette, France (e-mail: pierre.manil@cea.fr).

Color versions of one or more of the figures in this paper are available online at http://ieeexplore.ieee.org.

Digital Object Identifier 10.1109/TASC.2010.2043083

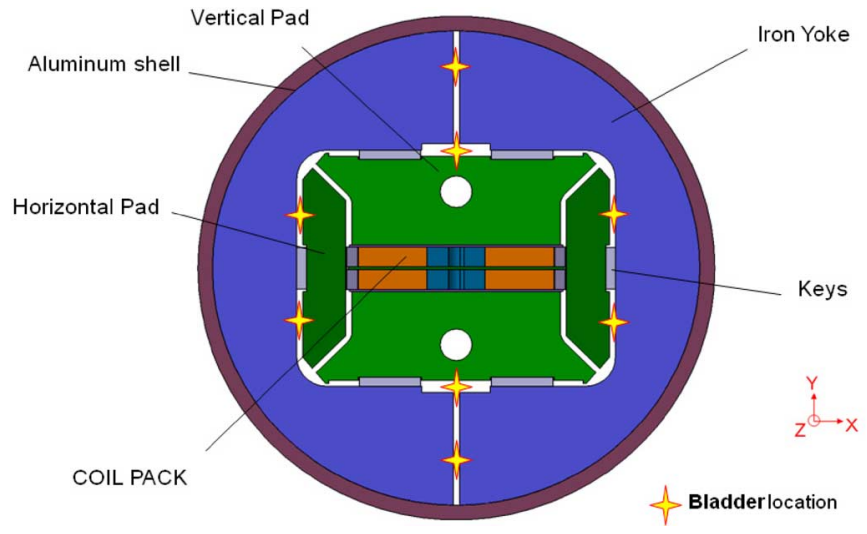

Fig. 1. Schematic view of the SMC cross section. The outer diameter is 540 $\mathrm{mm}$. The overall length measures $500 \mathrm{~mm}$.

pre-stress can be applied to the coil. Varying the pre-load, it will be so possible to both explore the mechanical limit of the $\mathrm{Nb}_{3} \mathrm{Sn}$ before degradation, and the impact of a low pre-stress on the magnet training performance. The SMC takes over the same design principles as the SD01 [6], a racetrack dipole coil tested at LBNL. This magnet showed very good performance, reaching $95 \%$ of the short sample current. Nevertheless some improvements were suggested:

- To re-design the coil in order to match the peak field region with the high stress one. This was not possible in SD01 since the peak field occurred in the coil ends, whereas the maximum stress was applied along the straight section.

- To improve the coil instrumentation, both for mechanical and magnetic analysis purposes.

The magnetic design of the SMC has been reported in [7]. The improvement mainly consisted in adopting coil end spacers in order to move the peak magnetic field along the coil straight section. In this paper we describe the mechanical optimization of the magnet structure, based on the optimized magnetic configuration. The mechanical design has been developed using the FEM codes ANSYS (CERN) and CAST3M (CEA) [8] in order to explore all the design parameters before converging to the final cross-checked configuration.

\section{Mechanical Structure}

The magnet structure is represented in Fig. 1. The SMC structure derives directly from the SD01's.

The structure has to accomplish three requirements: (1) to allow varying independently the lateral and longitudinal preloads on the coil; (2) to allow testing different cable and insulation types, and (3) to be easy to assemble and disassemble. The coil pack (coil and pads) is inserted into the magnet pack, 
TABLE I

MATERIAL PROPERTIES

\begin{tabular}{lcccc}
\hline \hline \multirow{2}{*}{ Component } & \multirow{2}{*}{ Material } & \multicolumn{2}{c}{$\mathrm{E}(\mathrm{GPa})$} & $\alpha \Delta \mathrm{T}$ \\
& & $293 \mathrm{~K}$ & $4.2 \mathrm{~K}$ & $(\mathrm{~mm} / \mathrm{m})$ \\
\hline Insulated conductor & $\mathrm{Nb}_{3} \mathrm{Sn}$ & 30 & 42 & 3.9 \\
Coil components & $\mathrm{Ti} \mathrm{A} 14 \mathrm{~V}$ & 110 & 130 & 1.71 \\
Shell/Rods & $\mathrm{A}$ 2014 T651 & 70 & 80 & 3.96 \\
Magnetic pad/yoke & MAGNETIL $^{\circledR}$ & 205 & 200 & 1.97 \\
Non-magnetic pads/yoke & AISI 316 LN & 210 & 193 & 2.6 \\
\hline \hline
\end{tabular}

formed by yoke and shell. The vertical pads are split into three parts, with a central made of magnetic iron (low-carbon steel MAGNETIL) and two outer end parts in stainless steel. The horizontal pads are machined out in one stainless steel piece. The magnet pack is an assembly of two iron halves surrounded by an aluminum shell. The lateral pre-load at Room Temperature (RT) is provided by using the bladders and keys technology [9]: the bladders, placed between the yoke and the coil pack, are inflated with pressurized water in order to create a clearance to insert the keys and corresponding shims. These shims, having different thickness, are used to set up the correct pre-stress once the bladders are deflated and removed. The longitudinal pre-stress at RT is provided by a couple of aluminum rods, tightened at one dipole end by means of a hydraulic piston. The final pre-stress is reached at cryogenic temperature, by the differential thermal contraction of the aluminum shell and rods with respect to the enclosed structure.

\section{2D MECHANICAL ANALYSIS}

The 2D mechanical analysis includes three main steps: (i) assembly with lateral pre-stress, (ii) cool down at $4.2 \mathrm{~K}$, and (iii) powering at short sample current. The 2D models are developed in order to set the proper lateral pre-load on the coil. In particular, it will be described how the pre-stress can be managed between warm and cryogenic conditions by changing the keys and the shell thickness. All the magnet parts are designed so to fulfill the failure criteria associated with the corresponding material, both at room and cryogenic temperature. The isotropic material properties for the magnet components are listed in Table I [10].

\section{A. ANSYS Model}

The 2D ANSYS model is represented in Fig. 2. 4-nodes planar elements are used, with plain stress option. Frictionless contact elements connect the different parts. A preliminary analysis of the appropriate coil pre-stress during assembly has been carried out. The lateral assembly interference $i_{x}$, reproducing the shim thickness, ranges from 100 to $1000 \mu \mathrm{m}$, in steps of $200 \mu \mathrm{m}$. We aim at verifying which is the maximum pre-load we can provide at RT in order to respect the Von Mises equivalent stress $\sigma_{\text {eqv }}=150 \mathrm{MPa}$ on the coil at short sample. The analysis reveals that the maximum lateral interference is about $400 \mu \mathrm{m}$ (see Fig. 3) from nominal key position. For higher interferences, the maximum stress on coil goes beyond the safe limit of $150 \mathrm{MPa}$. For very low interferences, the coil could detach from the central pole due to the Lorentz forces pull-out.

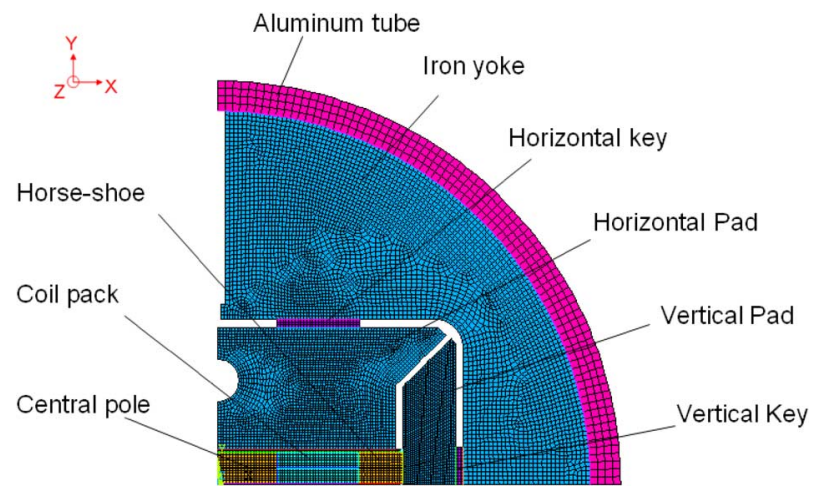

Fig. 2. 2D Mechanical model (ANSYS). The central pole and the horse shoe compose the coil components.

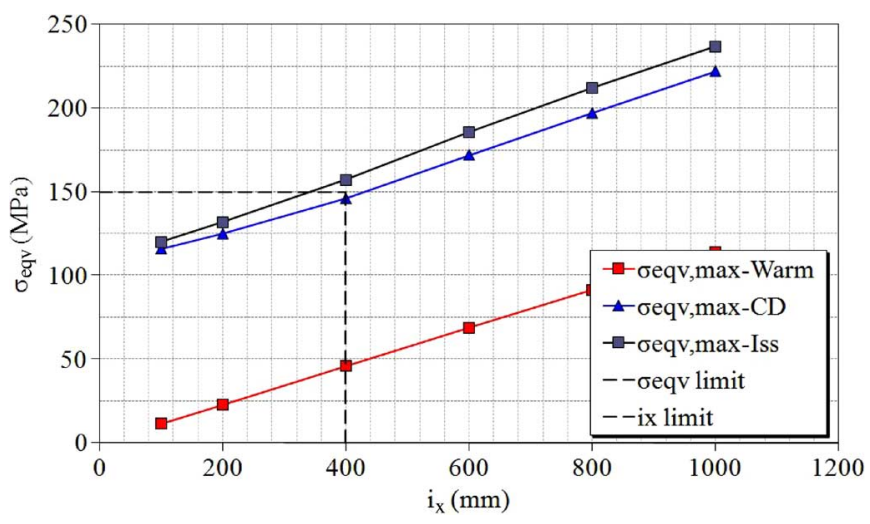

Fig. 3. $\sigma_{e q v, \max }$ on coil as a function of the assembly lateral interference $i_{x}$ For $i_{x}=400 \mu \mathrm{m}, \sigma_{e q v, \max }=155 \mathrm{MPa}\left(w_{y o k e}=55 \mathrm{~mm}\right)$.

This effect can be quantified in terms of maximum tensile stress on the epoxy impregnating the cables. This limit has to be $\leq 20 \mathrm{MPa}$ [11], according to experimental tests on $\mathrm{Nb}_{3} \mathrm{Sn}$ magnets.

For the previous analysis, the shell thickness has been arbitrarily set to $20 \mathrm{~mm}$. Nevertheless, the coil pre-load mainly derives from the combination of the assembly interference $i_{x}$, of the shell thickness $t_{\text {shell }}$, and of the yoke thickness $w_{y o k e}$. The relationship between the yoke thickness and the shell thickness is evaluated for several assembly interferences.

The curves shown in Fig. 4 are obtained by imposing three different design criteria: (i) the residual tensile stress between the coil and the central pole has to be lower than $20 \mathrm{MPa}$, (ii) $\sigma_{e q v, \max } \leq 150 \mathrm{MPa}$ on coil, and (iii) the maximum stress in the structure components has be lower than the equivalent mechanical limit with a safety factor (s.f.) of 1.5 . All the materials selected feature ductile behavior both at RT and $4.2 \mathrm{~K}$, exception made for the magnetic iron, which experiences brittle behavior at cryogenic temperature. For the first, the Von Mises criterion is assumed; whereas for MAGNETIL, the Rankine criterion based on the maximum principal stress is assumed. These curves represent the boundary between: (below) an insufficient loaded system, involving coil detachment at powering, and (above) an over constrained coil with $\sigma_{e q v, \text { max }}>150 \mathrm{MPa}$. Generally, the highest the pre-load, the thinner is the shell to be used. It also appears that an increase in yoke thickness up to $55 \mathrm{~mm}$ leads to higher pre-stress at RT, due to the augmented system rigidity, 


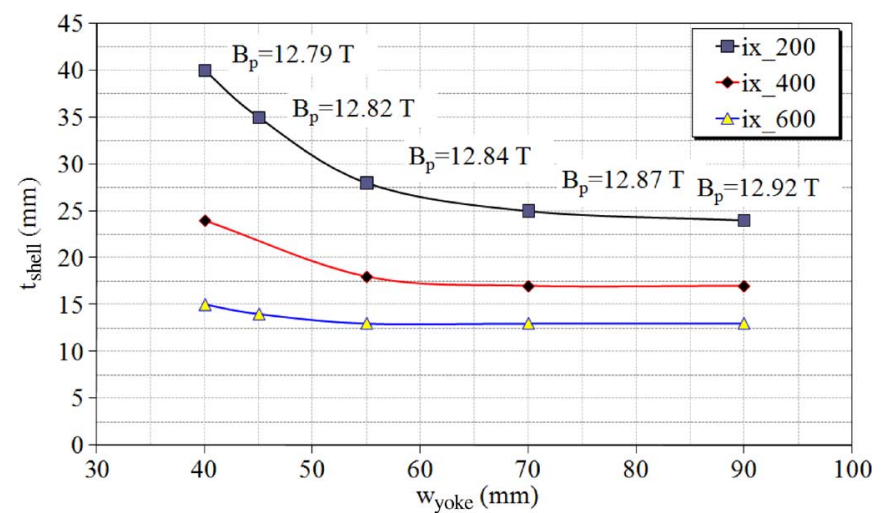

Fig. 4. Shell thickness satisfying the design constraints as a function of the yoke width $w_{y o k e}$ for different assembly interferences $i_{x}$. For every yoke thickness, the corresponding peak field on coil is shown.

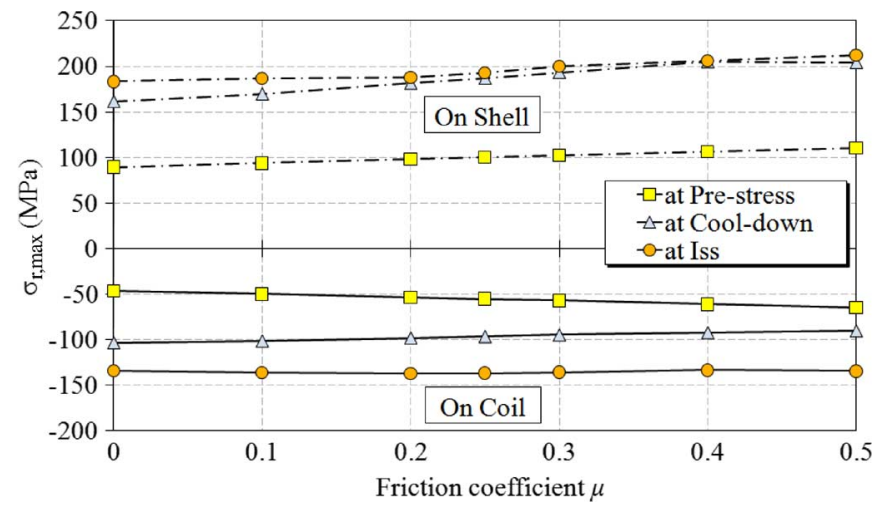

Fig. 5. Influence of the friction coefficient $\mu$ on the radial stress on the shell and coil. $w_{y o k e}=67 \mathrm{~mm}, t_{\text {shell }}=20 \mathrm{~mm}, i_{x}=0 \mu \mathrm{m}$.

so that a thinner shell can be used. For thicker yoke widths, the pre-load is then completely determined by the shell and interference dimensions. For a given magnet configuration, the pre-load will be tuned by means of the interference shims, according to the analysis purposes.

\section{B. CAST3M Model}

The effects of the friction coefficient in the magnet structure have been evaluated with a 2D model built in CAST3M. Fig. 5 represents the influence of the friction coefficient $\mu$ on the peak stress on coil and shell, at every simulation step.

We assume the same design criteria mentioned in the previous section. It appears that, for $i_{x}=0$ (i.e. no pre-load at RT), friction slightly affects the peak stress in the coil and in the shell, as well as it has second order effect on the related stress distribution. According to this, a frictionless model could effectively be used as a preliminary assessment of the mechanical behavior.

In reality, the effect of friction could be more important with higher lateral pre-load. Further study will be carried out once the experimental results will be available. After several iterative analyses, the shell thickness has been finally set to $20 \mathrm{~mm}$, the yoke thickness $w_{y o k e}$ to $90 \mathrm{~mm}$, and the assembly lateral interference at nominal conditions to $300 \mu \mathrm{m}$. In Fig. 6, the equivalent peak stress on coil, the average stress along the coil-pole side and coil-horseshoe side are represented for all the phases of the magnet life-cycle, up to $120 \%$ of the Lorentz forces.

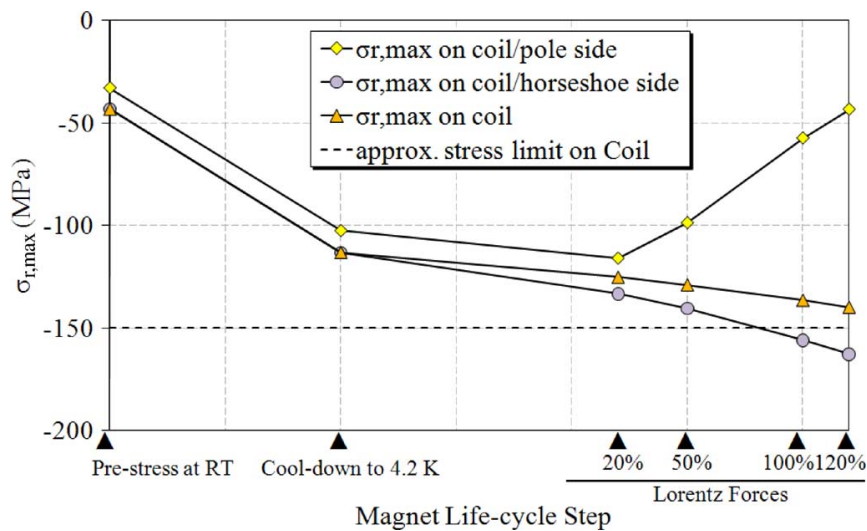

Fig. 6. Radial stress on coil during the magnet operating cycle, from assembly up to $120 \%$ of the Lorentz forces. $w_{y o k e}=90 \mathrm{~mm}, t_{\text {shell }}=20 \mathrm{~mm}, i_{x}=$ $300 \mu \mathrm{m}, \mu=0$.

Note that the horseshoe side undergoes stress increase due to Lorentz forces, which unload the main pole side at the same time, but keeping well below the tensile stress $\leq 20 \mathrm{MPa}$. At Iss the peak stress occurs inside the conductor block, reading about $150 \mathrm{MPa}$.

\section{3D MECHANICAL ANALYSIS}

The 3D analysis firstly aims at validating the results obtained with the 2D model, together with a detailed stress analysis of the magnet structure items.

The longitudinal pre-load system has then been set-up so to counterbalance the Lorentz forces effect tending to open up the coil at powering. Before launching a full 3D optimization, a preliminary study has been carried out in CAST3M on the coil pack model only (see Fig. 7). This analysis reveals that the horseshoe layout has almost no impact on the stress distribution in the coil ends and spacers. It will be machined out in one block, instead of separated pieces, for cost reasons. It also justifies that a longitudinal loading system is mandatory. The full 3D model has been built in ANSYS (Fig. 8). Since the coil impregnation glues the coil to the support structure, it was assumed bonding contact between elements of the coil-pack. The contact elements used elsewhere have been considered frictionless. Later modifications or improvements of the contact status will be done once the magnet will be tested. For this optimization, the rod diameter has been set to $28 \mathrm{~mm}$. The optimization process has been based on the radial stress component $\sigma_{r}$ at the boundary between the first cable turns and the central pole, being the point of the highest stress level eventually leading to coil motion [12]. The longitudinal pre-load is the input parameter; it is expressed in terms of rod displacement $d z(\mathrm{~mm})$ at RT, induced by the hydraulic piston.

Fig. 9 shows that the minimum displacement to be provided is about $1.5 \mathrm{~mm}$, independently from the lateral interference. This is due to the invariance of the lateral pre-load achieved, being the shell thickness adapted to any of the given assembly configurations, according to the results shown in Fig. 4.

For the final configuration, a rod diameter of $30 \mathrm{~mm}$ has been selected, and a longitudinal rod displacement $d z=1.5 \mathrm{~mm}$, corresponding to a tightening tension $\sigma_{z}=260 \mathrm{MPa}$. The choice of $30 \mathrm{~mm}$ has been done to further increase the safety margin 


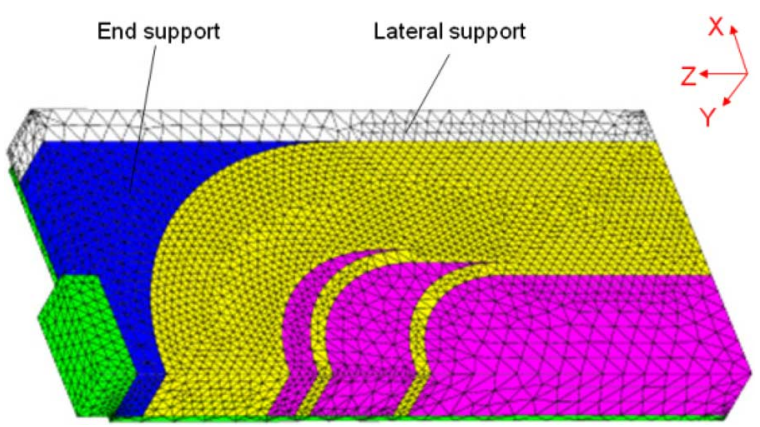

Fig. 7. Coil pack model (CAST3M). The horseshoe components, composed by the end and lateral support, are represented in blue and white respectively.

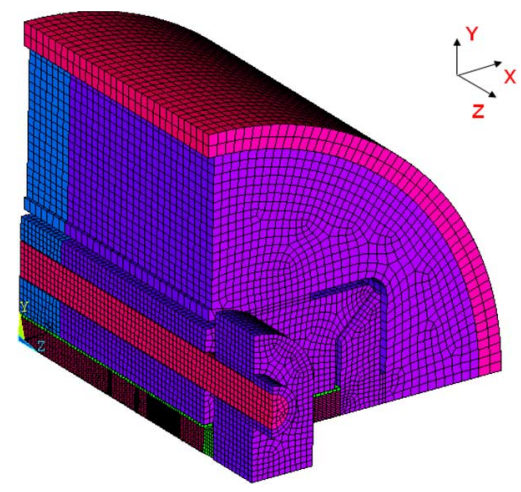

Fig. 8. 3D model of the SMC (ANSYS).

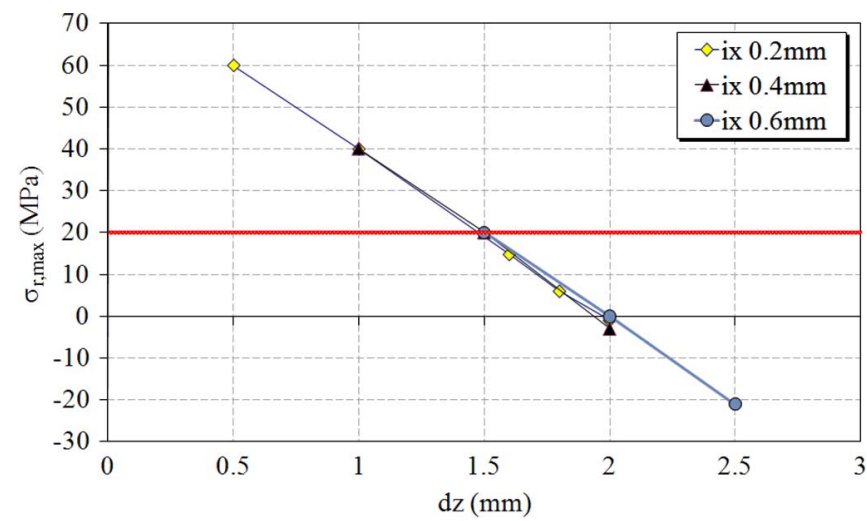

Fig. 9. $\sigma_{r, \max }$ on the inner coil end as a function of the longitudinal pre-load displacement $d z$, for different lateral assembly interferences.

on yield strength achieved with $28 \mathrm{~mm}$ diameter rods. A detailed stress analysis has been done on every magnet components during the four main steps of the magnet operating cycle, both in ANSYS and CAST3M. The main ANSYS results concerning the coil and the aluminum components, as active part of the pre-load system, are reported in Table II. At powering ( $I s s \approx 14 \mathrm{kA}$ ), the stress on coil is below the assumed safe limit of $150 \mathrm{MPa}$. The value of $190 \mathrm{MPa}$ after cool down might not be of real concern, since the magnet is not powered, if the coil behaves reversibly. In the aluminum components, the equivalent peak stress as well as the stress distribution does not vary from cool down to powering. This outlines that the pre-load parameters balance the magnetic forces preventing the coil from separation at the boundary with the central pole. The magnet structure
TABLE II

3D Model MaIN RESUlts

\begin{tabular}{cccccc}
\hline \hline Item & $\sigma(\mathrm{MPa})$ & $\begin{array}{c}\text { Axial } \\
\text { pre-load }\end{array}$ & $\begin{array}{c}\text { Lateral } \\
\text { pre-load }\end{array}$ & $\begin{array}{c}\text { Cool } \\
\text { down }\end{array}$ & Iss \\
\hline Coil & $\sigma_{V M, \max }$ & 57 & 38 & 190 & 130 \\
Sb $_{3}$ Sn limit & $\sigma_{\text {lim }}$ & 150 & 150 & 150 & 150 \\
\hline Shell & $\sigma_{V M, \max }$ & 6 & 60 & 140 & 140 \\
Rod & $\sigma_{V M, \max }$ & 270 & 280 & 430 & 430 \\
Al 2014 limit & $\sigma_{\text {lim }}$ & 415 & 415 & 545 & 545 \\
\hline \hline
\end{tabular}

fulfills the assumed failure criteria both at RT and $4.2 \mathrm{~K}$, with a safety factor of 1.5 . The rods are verified with s.f. $=1.3$.

\section{CONCLUSIONS}

The mechanical design of the SMC magnet has been presented. We described the procedure followed by the working group in order to perform a complete study of the design parameters. The required pre-stress on the coil has been set-up, in order to avoid any cable motion during powering, under the effect of Lorentz forces. A sensitivity analysis on the lateral pre-stress parameters has been performed, outlining how the shell thickness and the assembly interference interact. It has been remarked that the yoke plays a mechanical role up to a thickness of $55 \mathrm{~mm}$, a thicker yoke having an influence on the magnetic results only. The 3D model allowed setting up the longitudinal pre-stress. The rod pre-tension at RT has been evaluated in terms of end-plate displacement. Some tests of the mechanical structure are ongoing; they aim at verifying the overall behavior at RT and $4.2 \mathrm{~K}$ by means of an instrumented aluminum dummy coil-pack. At the same time, a test of the instrumentation will be performed. Test of the first full magnet will take place early 2010.

\section{REFERENCES}

[1] A. Lietzke et al., "Test results of $\mathrm{RD} 3 \mathrm{c}, \mathrm{a} \mathrm{Nb} \mathrm{Nb}_{3} \mathrm{Sn}$ common-coil racetrack dipole magnet," IEEE Trans. Appl. Supercond., vol. 13, pt. 2, pp. 1292-1296, Jun. 2003.

[2] S. Mattafirri et al., "Performance analysis of HD1: A $16 \mathrm{~T} \mathrm{Nb}_{3} \mathrm{Sn}$ dipole magnet," IEEE Trans. Appl. Supercond., vol. 15, pt. 2, pp. 1156-1159, Jun. 2005.

[3] S. Caspi et al., "Test results of $\mathrm{LARP} \mathrm{Nb}_{3} \mathrm{Sn}$ quadrupole magnets using a shell-based support structure (TQS)," IEEE Trans. Appl. Supercond., vol. 19, no. 3, pt. 2, pp. 1221-1225, Jun. 2009.

[4] A. Zlobin et al., "R\&D of $\mathrm{Nb}_{3} \mathrm{Sn}$ accelerator magnets at Fermilab," IEEE Trans. Appl. Supercond., vol. 15, pt. 2, pp. 1113-1118, Jun. 2005.

[5] P. Fessia, F. Regis, and E. Todesco, "Parametric analysis of forces and stresses in superconducting dipoles," IEEE Trans. Appl. Supercond., vol. 19, no. 3, pt. 2, pp. 1203-1207, Jun. 2009.

[6] H. Felice et al., "Design and test of a $\mathrm{Nb}_{3} \mathrm{Sn}$ subscale dipole magnet for training studies," IEEE Trans. Appl. Supercond., vol. 17, pt. 2, pp. 1144-1148, Jun. 2007.

[7] P. Manil et al., "Magnetic design and code benchmarking of the SMC (Short Model Coil) dipole magnet," IEEE Trans. Appl. Supercond., submitted for publication.

[8] [Online]. Available: www-cast3m.cea.fr..

[9] S. Caspi, "The use of pressurized bladders for stress control of superconducting magnets," IEEE Trans. Appl. Supercond., vol. 16, pt. 2, pp. 358-361, Jun. 2006.

[10] P. Loveridge, F. Regis, and P. Manil, "Mechanical Properties for NED SMC Mechanical Computations," CERN, Technical Report, 2007.

[11] P. Ferracin, Private Communication. April 2007, LBNL (USA).

[12] S. Caspi and P. Ferracin, "Toward integrated design and modeling of high field accelerator magnets," IEEE Trans. Appl. Supercond., vol. 16, pt. 2, pp. 1298-1303, Jun. 2006. 